\title{
Study on Coverage of Measles Rubella Vaccine at Natore Sadar Upazila and Awareness of the Mothers Regarding the Vaccine
}

\author{
Md. Belal Hossain, ${ }^{1}$ Md. Jawadul Haque ${ }^{2}$
}

\begin{abstract}
The present study was carried out with a view to explore the proportion of mothers vaccinated by Measles Rubella (MR) vaccine and to assess their knowledge level regarding MR vaccine in Natore Sadar Upazila. This study was a cross-sectional type of descriptive study. A total 153 mothers were selected purposively as respondents. Of them 140 were pregnant.

The present study showed that $40 \%$ of the respondents were in the age group of $20-24$ years with mean age of the respondents being $25.2 \pm 4.5$ years. The respondents were predominantly Muslim (94.1\%), literate (96.1\%) pregnant (91.5\%) and belonged to nuclear family (55.8\%). Over three-quarters (75.2\%) of the respondents had monthly family income of up to Taka 10,000 with average monthly income being Tk. $9975 \pm 9112$. Out of 153 respondents, about $44 \%$ were farmer. The respondents were invariably familiar (94.8\%) with MR vaccine and were aware about the total number of MR vaccines to be received to develop full immunity against measles and rubella. Majority (91.5\%) of their children received MR vaccine. Over $80 \%$ of the respondents knew the dose schedule of MR vaccine. However, approximately $95 \%$ of them did not know that MR vaccine is required during pregnancy. As association of demographic characteristics of the respondents with their level of knowledge on MR vaccine was analyzed, none of the demographic characteristics (age, educational status of the respondents, husband's occupation and monthly income) were found to be associated with their knowledge level $(p>0.05)$.

The study concluded that mothers, particularly pregnant mothers, know that there is a vaccine known as MR, but majority of them do not have adequate knowledge about target groups of this vaccine. This gap in knowledge should be minimized through health education to make wider coverage of the vaccine.
\end{abstract}

Key word: Coverage, Measles Rubella Vaccine, Awareness, Mothers

TAJ 2019; 32: No-1: 01-08

\section{Introduction}

Vaccines are among the most cost-effective interventions against infectious diseases. Immunization is one of the most successful public health initiatives in many countries including Bangladesh, yet continuous monitoring of coverage rates is essential to ensure high uptake for sustained success. ${ }^{1}$ EPI or expanded program on immunization, started in Bangladesh in 1979 as a pilot project to control or eliminate some vaccine preventable diseases. But coverage was below 2\% until 1985. Then, Government of Bangladesh started expanding the program in phases and brought whole country under coverage of EPI by the end of $1989 .{ }^{2}$ Presently, a Bangladeshi kid receives vaccines against 9 diseases: Tuberculosis, Polio, Diphtheria, Pertussis, Tetanus, Hepatitis B, H. Influenza type B infection, Measles and 
Rubella. A woman of reproductive age (15-49 years) should receive TT vaccine and MR vaccine. Vaccines against Rubella and pneumococcal meningitis and pneumonia are the latest vaccines added to schedule. ${ }^{1}$ However, the reintroduction of measles-rubella combined (MR) vaccination has raised concerns about adverse events as well as immunogenicity related to booster immunization in subjects with naturally acquired immunity to measles or rubella. ${ }^{3}$

Measles is one of the most contagious diseases ever known and is an important cause of death and disability among children worldwide. Rubella can cause foetal death or severe birth defects. Both are easily preventable with vaccines. Because these diseases are so contagious, a majority of the target population must be immunized, to develop herd immunity in order to slow an outbreak and prevent an epidemic. To do so the GAVI Alliance supported the recent measles-rubella (MR) campaign in Bangladesh to immunize more than 52 million children - one-third of the population of Bangladesh - and boost the country's present measles coverage from 86 to $98 \% .{ }^{4}$ Unlike previous measles vaccination campaigns, this one provided simultaneous protection against rubella. In 2012, the GoB introduced the combined MR vaccine into its Expanded Programme on Immunization (EPI) schedule for routine vaccination. ${ }^{5}$ The MR campaign was the largest in the country's history and vaccinated children between nine months and 15 years of age. This increased age range (beyond 15 months of age) was based on routine surveillance by the MOHFW that showed about $80 \%$ of the country's measles and rubella cases were contracted by children up to 15 years old ${ }^{6}$ Rubella vaccine (RA 27/3) is highly effective and has resulted in elimination of rubella from the Western hemisphere and several European countries. The vaccine stops the transmission of rubella from mother to child during pregnancy and prevent children from being born with severe birth defects. It will also protect children against measles. ${ }^{7}$ The vaccine used by the Measles \& Rubella Initiative is verified to be safe and effective by the WHO. ${ }^{8}$
Immunization coverage is one of the best public health outcome and service indicators of any country for the last 100 years. Parental decision regarding immunization is very important to improve immunization coverage. Persisting heterogeneity in coverage is a major contributor to continued measles mortality, and a barrier to measles elimination and introduction of rubellacontaining vaccine. Geographical isolation plays a key role in determining vaccine coverage. However, eliminating geographical inequities alone cannot achieve thresholds for herd immunity, indicating that changes in delivery rate or age range of routine vaccination will be required. Measles vaccine coverage remains far below targets for herd immunity in many countries including Bangladesh and is likely to be inadequate for achieving rubella elimination. To achieve measles and rubella elimination, coverage needs to be further increased among children aged 1-4 years and in regions with lower coverage. ${ }^{9}$ The aim of this study was to explore the coverage of MR vaccine and to know the level of awareness of mothers about MR vaccine and its association with their children's immunization completeness. Our objective is to identify determinants of inequities in coverage, and how vaccine delivery must change to achieve elimination goals, which is a focus of the WHO Decade of Vaccines.

\section{Materials and Methods}

This cross-sectional study conducted at Northern University Bangladesh over a period of 8 months from May, 2014 to December, 2014. All the mothers living in Natore Sadar Upazila constituted study population. Of them 153 (140 pregnant and 13 non-pregnant mothers) were purposively included in the study who voluntarily consented to participate in the study. Data were collected using a semi-structured questionnaire containing the variables of interest. Sociodemographic characteristics which among others, included age, occupation, education, monthly income, type of family etc. Families were classified as nuclear family (parents or parent, either father or mother with one or more unmarried child) and extended family (Parents or parent with married or never married children with or without other relatives 
e.g., father-in-law, mother-in-law, uncle, aunt, etc. eating from the same kitchen). Materials return in kind or cash in exchange of goods and services earned by the person (respondent) only is known as personal income and by the household members is known as household income. Household income or total family income consists of total income of all the members of the family living in the same household and taking food from the same cooking pot. Data were analysed using SPSS (Statistical Package for Social Sciences), version 16.0. The test statistics used to analyze the data were descriptive statistics and Chi-square $\left(\chi^{2}\right)$ Test. The level of significance was set at $5 \%$ and $p<0.05$ was considered significant.

\section{Result}

\section{Demographic characteristics:}

Out of 153 respondents approximately $40 \%$ were in the age range of $20-24$ years and $30.1 \%$ in the age group of 25-29 years with mean age of the respondents being $25.20 \pm 4.57$ years. Majority (83.7\%) of the respondents was engaged in diverse type of occupations, $7.2 \%$ were day labourer, $5.9 \%$ service-holder. Over three-quarters (75.2\%) of the respondents had monthly family income of Taka $<10,000,20.9 \%$ Taka 10,00-20,000 and a few (3.9\%) had income of Taka 20000 or more. The average monthly family income was Tk. $9975 \pm 9112$. Majority (94\%) of the respondents was Muslim. Approximately 85\% of the respondents had 1 -2 children in the family and the rest (15\%) 3- 4 children. Farming was the predominant occupation (43.8\%) followed by business (24.2\%), service (16.3\%), day labour (9.8\%) and others (5.9\%) (Table No. 1). In terms of educational status about $60 \%$ were primary level educated and 33.3\% were SSC \& HSS level educated and a few were graduate and higher level educated and very few were illiterate (fig.1). Nuclear family comprised the main bulk followed by joint family (56.8\%) and extended family (5.88\%) (fig.2).

Table 1: Distribution of the respondents by their demographic characteristics

\begin{tabular}{lcc}
\hline \multirow{2}{*}{ Demographic characteristics } & \multicolumn{2}{c}{ Respondents } \\
\cline { 2 - 3 } Age* (years) & No & $\%$ \\
$\quad<20$ & 17 & 11.1 \\
$20-24$ & 61 & 39.8 \\
$25-29$ & 46 & 30.1 \\
$30-34$ & 22 & 14.4 \\
$35+$ & 7 & 4.6 \\
Occupation & & \\
Service & 9 & 5.9 \\
Day-labour & 11 & 7.2 \\
Business & 3 & 1.9 \\
House-hold work & 2 & 1.3 \\
$\quad$ Others & 128 & 83.7 \\
Religion & & \\
$\quad$ Muslim & 144 & 94.1
\end{tabular}


Hindu

Monthly income** (Taka)

$<10000$

$10000-20000$

20.9

$\geq 20000$

6

Number of children

$$
\begin{aligned}
& 1-2 \\
& 2-3
\end{aligned}
$$

\section{Husbands' Occupation}

Farmer

Business

Service

Day labour

$*$ Mean \pm SD $=25.2 \pm 4.5$ years; $* *$ Mean \pm SD $=(9975 \pm 9112)$ Taka.

Distribution of respondents by educational status

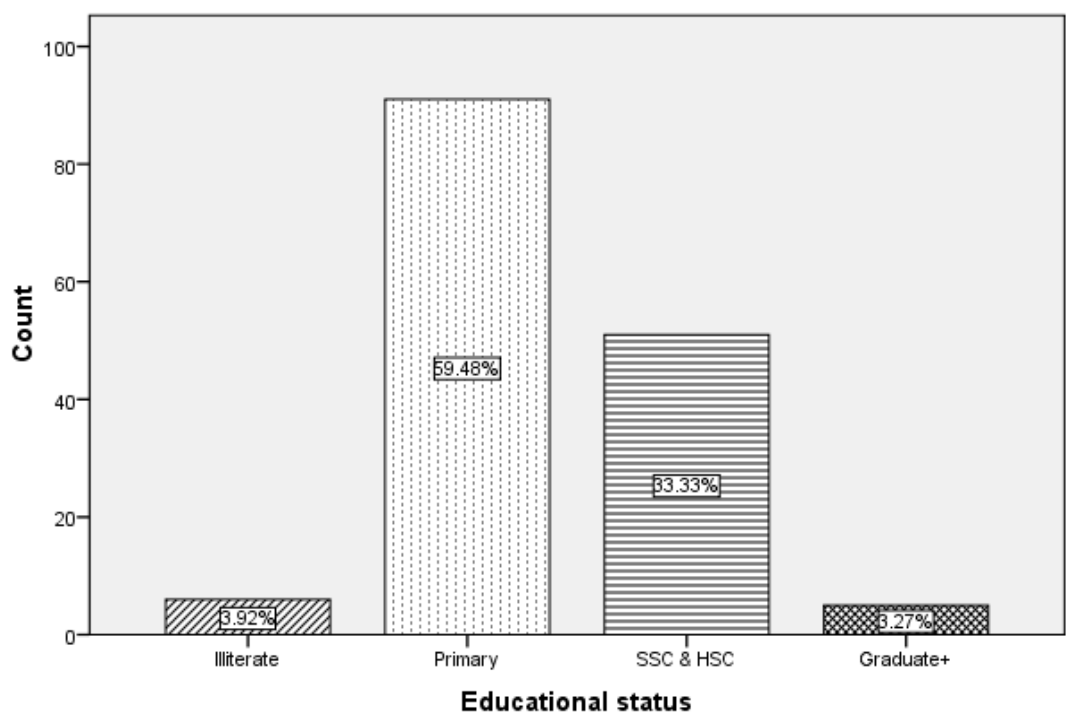

Fig. 1: Distribution of respondents by their educational status $(n=153)$ 


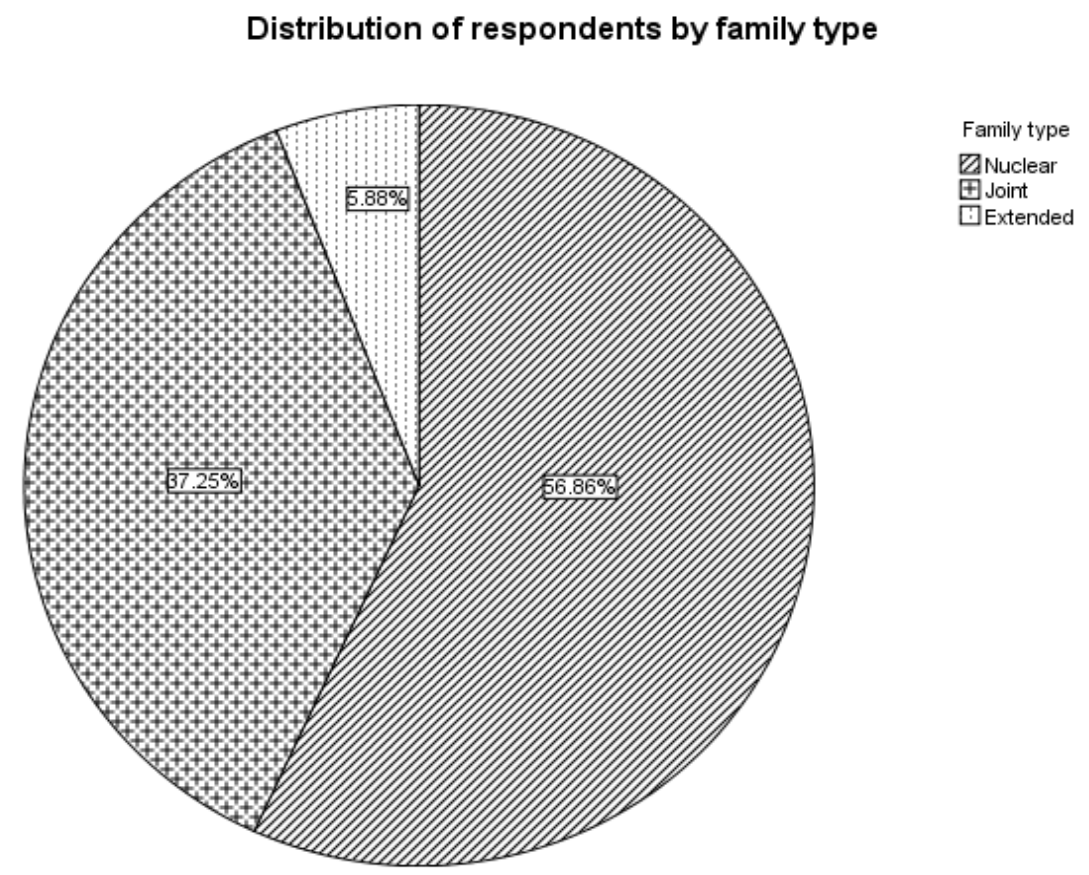

Fig. 2: Distribution of respondents by their family type $(n=153)$

\section{Respondents' knowledge about MR vaccine:}

Over $90 \%$ of the respondents were informed about the MR vaccine and that the vaccine is given to children. Asked about the target people of MR vaccine, $89.5 \%$ correctly mentioned the target group. Majority (83.7\%) also knew the dose-schedule of the vaccine. However, only a few (5.2\%) had the knowledge that MR vaccine is required during pregnancy (Table II).

Table II: Distribution of the respondents by their knowledge about MR vaccine

\begin{tabular}{lcc}
\hline & \multicolumn{2}{c}{ Respondents } \\
\cline { 2 - 3 } Knowledge about MR vaccine & No & $\%$ \\
\hline Perception about MR vaccine & 140 & 91.5 \\
Yes & 13 & 8.5 \\
No & 140 & 91.5 \\
MR vaccine could be given to children & 13 & 8.5 \\
$\quad$ Service & & \\
Day-labour & 137 & 89.5 \\
Target people for MR vaccine & 16 & 10.5 \\
Yes & 128 & 83.7 \\
No & 25 & 16.3 \\
Know the dose-schedule of MR vaccine & & 5.2 \\
Yes & 8 & 94.8 \\
No & 145 & \\
MR vaccine needed during pregnancy & & \\
Yes & & \\
No & & \\
\hline
\end{tabular}


Respondents' knowledge about MR vaccine and demographic characteristics:

Of the 153 respondents, 135(88.2\%) had adequate knowledge about MR vaccine. Three demographic characteristics of the respondents (age, educational status and monthly income) one demographic characteristic of their husbands (occupation) were compared between respondents' level of knowledge on MR vaccine to find if there was any association of respondents' level of knowledge with their demographic characteristics. None of the four demographic characteristics were found to be associated with the respondents' level of knowledge on MR vaccine ( $p>0.05$ in each case) (table III)

Table III: Distribution of the respondents by their demographic characteristics

\begin{tabular}{|c|c|c|c|}
\hline \multirow{2}{*}{ Demographic characteristics } & \multicolumn{2}{|c|}{$\begin{array}{c}\text { Adequate knowledge about } \\
\text { MR vaccine }\end{array}$} & \multirow{2}{*}{$\chi^{2}$ (p-value) } \\
\hline & $\begin{array}{c}\text { Yes } \\
(\mathrm{n}=135)\end{array}$ & $\begin{array}{c}\text { No } \\
(\mathrm{n}=18)\end{array}$ & \\
\hline \multicolumn{4}{|l|}{ Age* (years) } \\
\hline$<30$ & $111(82.2)$ & $13(72.2)$ & \multirow{2}{*}{$0.485(0.486)$} \\
\hline$\geq 30$ & $24(17.8)$ & $5(27.8)$ & \\
\hline \multicolumn{4}{|l|}{ Educational status } \\
\hline Up to primary & $85(63.0)$ & $12(66.7)$ & \multirow{2}{*}{$0.094(0.759)$} \\
\hline SSC to higher & $50(37.0)$ & $6(33.3)$ & \\
\hline \multicolumn{4}{|l|}{ Monthly income** (Taka) } \\
\hline Very poor income $($ Taka $\leq 10000)$ & 99(73.3) & 16(88.9) & \multirow{2}{*}{$1.310(0.252)$} \\
\hline Poor to fair income (Taka > 10000) & $36(26.7)$ & $2(11.1)$ & \\
\hline \multicolumn{4}{|l|}{ Husbands' occupation } \\
\hline Farmer \& day labour & $72(53.3)$ & $10(55.6)$ & \multirow{2}{*}{$0.032(0.859)$} \\
\hline Business, service \& others & $63(46.7)$ & $6(44.4)$ & \\
\hline
\end{tabular}

Figures in the parentheses denote corresponding percentage. Data were analyzed using Chi-squared $\left(\chi^{2}\right)$ Test

\section{Discussion}

Immunization is one of the most successful public health initiatives in Canada, yet continuous monitoring of coverage rates is essential to ensure high uptake for sustained success. This crosssectional type of descriptive study which was carried out with the objective of exploring to proportion of mothers vaccinated by MR vaccine and to explore knowledge of the mothers regarding MR vaccine in Natore Sadar Upazila.

Measles is one of the most contagious diseases ever known and is an important cause of death and disability among children worldwide. Vaccination against measles was already in EPI schedule. In 
2012, the Go B introduced the combined MR vaccine into its Expanded Programme on Immunization (EPI) schedule for routine vaccination. A woman of reproductive age (15-49 years) should receive TT vaccine as well as MR vaccine. ${ }^{11}$ Rubella can cause foetal death or severe birth defects. Both are easily preventable with vaccines. Because these diseases are so contagious, a majority of the population (90-95\% in this case) must be immunised in order to slow an outbreak and prevent an epidemic. To achieve "community" or "herd" immunity in Bangladesh, the GAVI Alliance supported the recent measlesrubella (MR) campaign to immunise more than 52 million children---one third the population of Bangladesh---and boost the country's present measles coverage of $86 \%$ up to $98 \%$.

The MR campaign was the largest in the country's history and vaccinated children between nine months and 15 years of age. This increased age range (beyond 15 months of age) was based on routine surveillance by the MOHFW that showed about $80 \%$ of the country's 2011 measles and rubella cases were contracted by children up to 15 years old. The Go B is planning a subsequent campaign to offer the crucial second dose of the MR vaccination shown to be key to prevention. Thousands of health personnel and community volunteers worked in more than 170,000 schools and 150,000 immunization centres to carry out the three-week MR campaign, with special teams covering urban public spaces and rural hard-toreach places. It was the largest-ever MR campaign launched to date with support from the GAVI Alliance---a public-private global health partnership committed to increasing access to immunization in poor countries. ${ }^{6}$

In the present study nearly $90 \%$ of the mothers had adequate knowledge on MR vaccine. Majority (91.5\%) of the respondents knew that the target people for MR vaccination are children and the number of doses to be given is two. However, only a few of them (5.2\%) were aware that the vaccine is required during pregnancy. Majority $(88.2 \%)$ of the mothers had adequate knowledge about MR vaccine. However, none of the four demographic characteristics analyzed were found to be associated with the respondents' level of knowledge on MR vaccine. The 2012 SIA successfully increased MR coverage; however, to maintain measles and rubella elimination, coverage needs to be further increased among children aged 1-4 years and in regions with lower coverage. ${ }^{10}$ In 2008, following a rubella epidemic, the Egyptian Ministry of Health implemented a Measles Rubella (MR) catch-up campaign, based on WHO recommendations for supplementary immunization activities to eliminate measles by 2010. The age group targeted was 10-20 years. This campaign was unique in Egypt as it was the first national vaccination campaign which included university students. To report uptake of MR vaccine and reasons for declining the vaccine among medical and non-medical students in the campaign and to assess the knowledge about the vaccine and the diseases. The study was conducted in two stages. In the first stage during the vaccination sessions, medical and other students were given a questionnaire to assess their knowledge of the campaign and the vaccine. The second stage was carried out when the campaign was completed to assess vaccine uptake among medical students. Posters displayed inside the university were reported to be the main source of information about the campaign. Students were generally poorly informed about both vaccine adverse effects, and contraindications although medical students tended to be better informed than other students. Overall $64.8 \%$ medical students accepted the vaccine with higher uptake among females than males (85.9 and 58.3\% respectively). Non-compliant students had a significantly higher mean age. Almost half of students who did not accept the vaccine gave their reason as having little information about the vaccine (43.3\%). The MR campaign in Ain Shams University may have been more successful with better use of health education messages. ${ }^{11}$

The reported coverage of the measles-rubella (MR) or measles-mumps-rubella (MMR) vaccine is greater than $99.0 \%$ in Zhejiang province. However, the incidence of measles, mumps, and rubella remains high. The study assessed MMR seropositivity and disease distribution by age on 
the basis of the current vaccination program, wherein the first dose of MR is administered at 8 months and the second dose of MMR is administered at 18-24 months. Cross-sectional serological surveys of MMR antibodies were conducted by collecting epidemiological data in Zhejiang province, China in 2011. In total, 1015 participants were randomly selected from two surveillance sites. Serum MMR-specific immunoglobulin $\mathrm{G}$ levels were tested by enzymelinked immunosorbent assay. A timely two-dose MMR vaccination schedule is recommended, with the first dose at 8 months and the second dose at 18-24 months. An MR vaccination speed-up campaign may be necessary for elder adolescents and young adults, particularly young females. ${ }^{10}$

\section{Conclusion}

The study concluded that most of the mothers had adequate knowledge on MR vaccine. They knew that the target people for MR vaccination are children and the number of doses to be given to immunize a child completely against Measles and Rubella are two. However, only a negligible proportion of them were aware that the vaccine is required during pregnancy. Majority of the mothers had adequate knowledge about MR vaccine. None of the demographic characteristics of the mothers were found to be associated with the respondents' level of knowledge on MR vaccine.

\section{References}

1. Kizito D, Tweyongyere R, Namatovu A, Webb EL, Muhangi L, Lule SA, et al. Factors affecting the infant antibody response to measles immunisation in Entebbe-Uganda. BMC Public Health. 2013; 13: 619.

2. Lin $\mathrm{W}$, Xiong $\mathrm{Y}$, Tang $\mathrm{H}$, Chen $\mathrm{B}$, Ni J. Factors associated with delayed measles vaccination among children in Shenzhen, China: A case-control study. Hum Vaccin Immunother. 2014; 10(12): 3601-6.

3. Marin M, Broder KR, Temte JL, Snider DE, Seward JF. Centers for Disease Control and Prevention (CDC). Use of combination measles, mumps, rubella, and varicella vaccine: recommendations of the Advisory Committee on Immunization Practices (ACIP). MMWR Recomm Rep. 2010; 59(RR-3):112.

4. Pearce $A$, Mindlin $M$, Cortina-Borja $M$, Bedford $H$. Characteristics of 5-year-olds who catch-up with MMR: findings from the UK Millennium Cohort Study. BMJ Open. 2013; 3(7).

5. Aaby P, Bhuiya A, Nahar L, Knudsen K, de Francisco A, Strong M. The survival benefit of measles immunization may not be explained entirely by the prevention of measles disease: a community study from rural Bangladesh. Int $\mathrm{J}$ Epidemiol. 2003; 32(1):106-16.

6. http://www.icddrb.org/component/content/article/10 030-news/4193-evaluating-the-measles-rubellavaccination-campaign-in-bangladesh Accessed on 14.03.2015

7. http://bdnews24.com/health/2014/01/20/measlesrub ella-vaccination-drive 14.03.2015

8. http://www.measlesrubellainitiative.org/learn/thesolution/the-vaccine/ Accessed on 14.03.2015

9. Tohme RA, François J, Wannemuehler K, Magloire $\mathrm{R}$, Danovaro-Holliday MC, Flannery $\mathrm{B}$, et al. Measles and rubella vaccination coverage in Haiti, 2012: progress towards verifying and challenges to maintaining measles and rubella elimination. Trop Med Int Health. 2014; 19(9):1105-15.

10. Wang Z, Yan R, He H, Li Q, Chen G, Yang S, et al. Chen E. Difficulties in eliminating measles and controlling rubella and mumps: a cross-sectional study of a first measles and rubella vaccination and a second measles, mumps, and rubella vaccination. PLoS One. 2014; 9(2):e89361.

11. Abd Elaziz K M, Sabbour SM, Dewedar SA. A measles and rubella (MR) catch-up vaccination campaign in an Egyptian University: Vaccine uptake and knowledge and attitudes of students. Vaccine. 2010;28(47):7563-68.

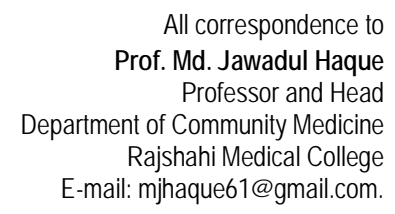

All correspondence to

Md. Jawadul Haque of Community Medicine mail: mjhaque61@gmail.com. 